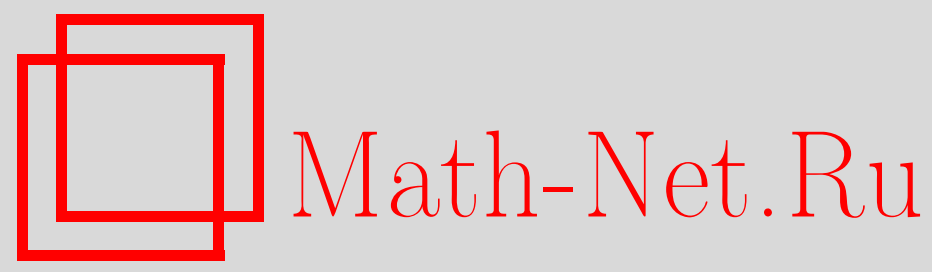

Н. В. Гравин, Невырожденные раскраски в теореме Брукса, Дискрет. матем., 2009, том 21, выпуск 4, 105-128

DOI: https://doi.org/10.4213/dm1076

Использование Общероссийского математического портала Math-Net.Ru подразумевает, что вы прочитали и согласны с пользовательским соглашением http://www . mathnet.ru/rus/agreement

Параметры загрузки:

IP: 52.6 .47 .48

26 апреля 2023 г., 12:09:13 


\title{
Невырожденные раскраски в теореме Брукса
}

\author{
(C) 2009 г. Н. В. Гравин
}

\begin{abstract}
Работа посвящена обобщению широко известной теоремы Брукса о невырожденной раскраске графов. Обобщение достигается за счет введения другого понятия невырожденности раскраски, при котором раскраска является невырожденной в обычном смысле не для всех вершин, а для вершин достаточно большой степени.
\end{abstract}

\section{1. Введение}

Мы будем следовать терминологии и обозначениям, взятыми из книги [1], и рассматривать только конечные графы без петель. Как и в [1], через $\delta(G)$ и $\Delta(G)$ будем обозначать минимальную и максимальную степени вершин графа $G$. Окрестностью вершины $v \in V(G)$ в графе $G$ называется множество

$$
N_{G}(v)=\{u \in V(G): u v \in E(G)\} .
$$

Вершины из $N_{G}(v)$ называются соседями $v$. Через $|S|$ будем обозначать мощность множества $S$, индуцированный подграф графа $G$ на множестве вершин $S \subseteq V(G)$ будем обозначать через $G(S)$, через $G-S-$ граф $G(V(G) \backslash S)$, а через $d_{G}(v)$ будем обозначать степень вершины $v$ в графе $G$.

Правильной раскраской графа $G$ называется отображение $c: V(G) \mapsto\{1,2, \ldots, k\}$ такое, что если $u, v \in V(G)$ смежны в $G$, то $c(u) \neq c(v)$. Пусть теперь $c-$ правильная раскраска $G$ в $k$ цветов, а множество $V^{\prime} \subseteq V(G)$, тогда через $c\left(V^{\prime}\right)$ мы будем обозначать ограничение отображения $c$ на множество $V^{\prime}$. Таким образом, мы получаем правильную раскраску графа $G\left(V^{\prime}\right)$ в $k$ цветов.

Настоящая работа посвящена обобщению широко известной теоремы Брукса [4], которая говорит, что хроматическое число $\chi(G) \leqslant \Delta(G)$ для любого графа $G$ без полных подграфов на $d+1$ вершине с $\Delta(G)>2$. В нашей работе дополнительно накладывается требование $(c, p)$-невырожденности на раскраску графа.

Раскраска называется $c$-невырожденной, если среди соседей любой вершины графа степени хотя бы $c$ встречаются вершины хотя бы $c$ различных цветов. Эта задача усиления теоремы Брукса имеет долгую историю. Так в работах [2, 3] предпринимались попытки найти оценки на числа цветов, в которое можно правильным невырожденным образом покрасить граф в случае $c=2$. Однако полученные в этих работах результаты не являются обобщениями теоремы Брукса, так как число цветов, в которые мы красим граф, увеличивается по сравнению числом, указанным в теореме Брукса. Дальнейшие исследования, уже для произвольного значения $c$, можно найти в работах $[5,6]$. В этой же работе авторы замечают, что было бы интересно получить усиление теоремы Брукса в смысле невырожденности. Несмотря на значительный интерес к данной теме, задача 
подобного обобщения теоремы Брукса до сих пор не решена и представляется слишком сложной, чтобы решать ее в подобной формулировке, то есть когда невырожденность раскраски накладывается на все вершины графа степени хотя бы $c$.

Приведем соображение, заставляющее задуматься о необходимости изменения постановки задачи. Действительно, наличие в графе вершин степени $c$ означало бы, что все ее соседи покрашены в различные цвета, и эту вершину можно было бы при тех же требованиях на граф заменить на $c$-гиперребро между всеми ее соседями, рассматривая теперь граф как граф с гиперребрами. Проведя данное преобразование для нескольких вершин степени $c$, мы можем получить произвольный граф с $c$-гиперребрами, а значит, и наши результаты обобщаются на подобные графы, которые являются весьма сложными объектами для исследования.

Ключевым соображением, позволяющим получить серьезные результаты в данной области, является то, что мы потребуем несколько другое условие невырожденности раскраски, которое назовем $(c, p)$-невырожденностью, а именно, чтобы раскраска была невырожденной в обычном смысле не для всех вершин, а для вершин достаточно большой степени.

Определение 1. Пусть даны натуральные числа $c \geqslant 2$ и $p \geqslant c$. Раскраска вершин графа $G$ называется $(c, p)$-невырожденной, если для любой вершины графа $G$ степени хотя бы $p$ среди ее соседей найдутся вершины хотя бы $c$ различных цветов.

Такое изменение условия невырожденности позволяет нам доказать следующую весьма общую теорему, а также получить в процессе доказательства несколько интересных следствий.

Теорема 1. Пусть дано число $D \geqslant 3$ и граф $G$ без клик на $D+1$ вершине такой, что степень любой его вершины не превосходит $D$. Тогда для любого $c \geqslant 2$ существует правильная (c, p)-невырожденная раскраска вершин графа $G$ в $D$ ияветов, где

$$
p=\left(c^{3}+8 c^{2}+19 c+6\right)(c+1) .
$$

Также во введении нельзя не упомянуть работу [7], где авторами получены сильные результаты. Сформулируем основной результат работы [7].

Определение 2. Раскраска графа называется $\beta$-экономной, если никакая вершина графа не имеет в своей окрестности более чем $\beta$ соседей одного цвета.

Теорема 2. Улюбого графа $G$ с максимальной степенью

$$
\Delta \geqslant \Delta_{0}=e^{10^{7}}
$$

сущзествует правильная $\left\lceil\log ^{8} \Delta\right\rceil$-экономная раскраска в $\Delta+1$ извет.

Из этой теоремы очевидным образом можно получить следующее утверждение.

Следствие 1. У любого графа G с максимальной степенью

$$
\Delta \geqslant \Delta_{0}=e^{10^{7}}
$$

сущеествует правильная $\left(\Delta_{0} /\left\lceil\log ^{8} \Delta_{0}\right\rceil, \Delta_{0}\right)$-невырожденная раскраска в $\Delta+1$ ичвет. 
Таким образом, можно получить оценку невырожденности раскраски асимптотически гораздо лучшую, чем полученная в этой статье, используя при этом всего один лишний цвет. Однако между правильной раскраской в $\Delta$ цветов и $\Delta+1$ цвет существует большая разница. Можно с легкостью рекурсивно покрасить граф в $\Delta(G)+1$ цвет, однако факт, что связный граф $G \neq K_{n}$ с $\Delta(G) \geqslant 3$ имеет правильную раскраску в $\Delta(G)$ цветов, является в точности одной из формулировок теоремы Брукса [4]. При этом также необходимо отметить, что это следствие, как и все результаты в работы [7], получены для очень больших графов. По сравнению с числами $\Delta \geqslant \Delta_{0}=e^{10^{7}}$, наша оценка

$$
p=\left(c^{3}+8 c^{2}+19 c+6\right)(c+1)
$$

представляется значительно меньшей. Она дает нам осмысленное усиление теоремы Брукса уже при $c=2$ и $p=252$, то есть уже для графов с $\Delta \geqslant 252$.

Существует интересное соображение, касающееся $\beta$-экономной и $(c, p)$-невырожденных раскрасок. Одним из объяснений, почему изучаются правильные раскраски графов, может служить то, что раскраска графа $G$ в $c$ цветов есть в точности гомоморфизм $G$ в граф $K_{c}$ (определение и более полная информация, касающаяся гомоморфизмов графов, содержится в книге [8]). Далее можно сделать естественный шаг и рассмотреть для каждой вершины графа множество ее соседей, а проще говоря, ее окрестность. Тогда условие $(c, p)$-невырожденности будет в точности означать, что для каждой вершины $v$ такой, что $\left|N_{G}(v)\right| \geqslant p$, размер образа есть не менее $c$, то есть $(c, p)$-невырожденность накладывает ограничение снизу на размер образа отображения, в то время как свойство, состоящее в том, что раскраска является $\beta$-экономной, будет устанавливать верхнюю оценку на размер ядра отображения, где под размером ядра естественно понимать максимальный размер подмножества окрестности, которое отображается в один цвет.

Одним из основных этапов доказательства теоремы 1 является доказательство следующей теоремы 2, которая сама по себе представляется весьма интересной.

Теорема 3. Пусть $G$ - граф без клик размера $D+1$ с максимальной степенью, не большей, чем D, и пусть, кроме того,

$$
D=\sum_{i=1}^{c+1} \alpha_{i}, \quad \alpha_{i} \geqslant 2
$$

Тогда в множестве $\Xi$ раскрасок графа $G$ в $c+1$ ивет найдется такая раскраска $\xi$, что

(1) $\Phi(\xi)=\min _{\psi \in \Xi} \Phi(\psi)$, гдe

$$
\Phi=\sum_{i=1}^{c+1} \frac{f_{i}}{\alpha_{i}}
$$

a $f_{i}$ - число ребер графа $G$, соединяющих две вершины иявета $i$,

(2) для любого і раскраска $\xi$ не содержит клик размера $\alpha_{i}+1$ на вершинах иявета $i$,

(3) $\Delta_{G\left(V_{i}\right)} \leqslant \alpha_{i}$, где $V_{i}-$ множество всех вершин иявета $i$ в графе $G$. 
В частности, одним из прямых следствий этой теоремы является результат, близкий по смыслу к приведенному в [9] и состоящий в том, что вершины любого графа $G$ с $\Delta_{G} \leqslant k$ можно разбить на $n$ множеств $V_{1}, V_{2}, \ldots, V_{n}$ таких, что $\Delta_{G\left(V_{i}\right)} \leqslant k_{i}$, где

$$
\sum_{i=1}^{n} k_{i}=k-n+1 .
$$

Следствие 2. Пусть $G$ - граф без клик размера $D+1$ с максимальной степенью, не большей $D, u$

$$
D=\sum_{i=1}^{k} \alpha_{i}, \quad \alpha_{i} \geqslant 2
$$

Тогда вершины графа $G$ можно разбить на $k$ непересекающихся множеств $V_{1}, V_{2}, \ldots, V_{k}$ таких, что для любого $i \in\{1,2, \ldots, k\}$ в графе $G\left(V_{i}\right)$ нет клик на $\alpha_{i}+1$ вершине и степени всех вершин в $G\left(V_{i}\right)$ не больше $\alpha_{i}$.

\section{2. Доказательство основной теоремы}

Замечание 1. Отметим, что $(c, p)$-невырожденность раскраски является весьма сильным условием, так как даже в случае двудольного графа $G$ не понятно, почему можно так раскрасить граф в $\Delta_{G}$ цветов. А если еще красить двудольный граф правильным образом в $D$ цветов с выполнением условия $(c, p)$-невырожденности, не ограничивая при этом максимальную степень этого двудольного графа, то теорема 1 становится просто неверной, причем уже для $c=2$ и любого значения $p$.

Приведем контрпример.

В качестве первой доли нашего двудольного графа $G$ возьмем множество $S_{1}$, состоящее из $(p-1) D+1$ элемента, в качестве второй доли возьмем множество всех $p$-элементных подмножеств множества $S_{1}$ и соединим каждую такую выборку со всеми входящими в нее элементами из $S_{1}$ (см. рис. 1). Попытаемся раскрасить получившийся двудольный граф в $D$ цветов. Заметим, что по принципу Дирихле в множестве $S_{1}$ при любой раскраске графа $G$ в $D$ цветов найдется $p$ вершин одного цвета, а значит соответствующая выборка во второй доли графа $G$ будет смежна с вершинами только одного цвета.

Замечание 2. К сожалению, оценка

$$
p(c)=\left(c^{3}+8 c^{2}+19 c+6\right)(c+1)
$$

для малых с дает достаточно большое значение. Вполне возможно, что с помощью нашего метода доказательства можно получить лучшую оценку. Однако нашим методом получить асимптотику лучшую, чем $c^{4}\left(1+O\left(c^{-1}\right)\right)$, не удается.

Теорема 4. Пусть $D \geqslant 3 u G=(V, E)-$ граф без клик на $D+1$ вершине такой, что степень любой его вершинь не превосходит $D$. Тогда для любого $c \geqslant 2$ существует правильная $(c, p)$-невырожденная раскраска вериин графа $G$ в $D$ ияветов, где

$$
p=\left(c^{3}+8 c^{2}+19 c+6\right)(c+1) .
$$




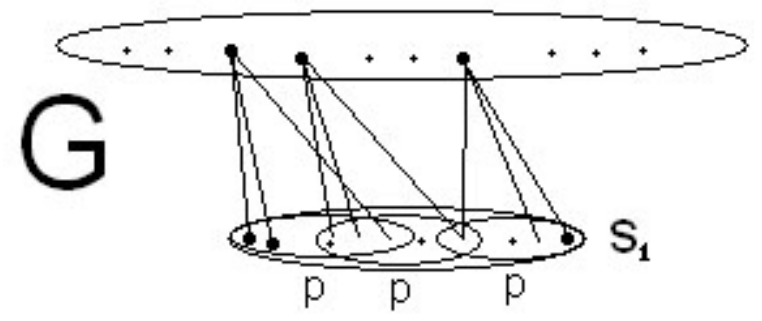

Pис. 1.

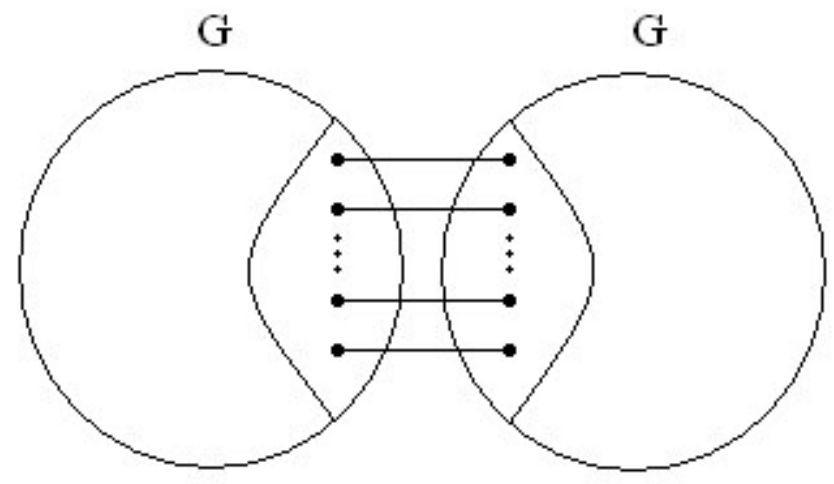

Pис. 2.

Предложение 1. Не умаляя общности, можно считать, что в графе $G$ нет вершин, степени которых меньше р.

Доказательство. С графом $G$ можно проделать следующую операцию: взять две копии графа $G$ и соединить в этих копиях все пары одинаковых вершин, степени которых меньше $p$ (см. рис. 2).

Заметим, что, для нового графа выполнены все условия теоремы 1. Также заметим, что, если удастся покрасить правильным образом новый граф в $D$ цветов так, что в окрестности каждой вершины степени хотя бы $p$ будет $c$ различных цветов, то обе копии графа $G$ будут автоматически раскрашены правильным образом в $D$ цветов с выполнением того же условия. Проделав такую операцию несколько раз, в итоге мы получим граф, в котором не будет вершин степени, меньшей $p$, ибо при каждой такой операции минимальная степень графа увеличивалась (если, конечно, в графе были вершины степени меньшей, чем $p$ ).

Доказательство теоремы 1 состоит из двух частей. В первой части мы сведем теорему к лемме 1, а во второй части мы докажем эту лемму. 


\section{3. Первая часть доказательства теоремы 1}

Покрасим граф $G$ произвольным образом в цвета $1,2, \ldots, c+1$. Поставим в соответствие каждому цвету $i \in\{1,2, \ldots, c+1\}$ число $\alpha_{i}$, где $\alpha_{i}=\lceil D /(c+1)\rceil$, либо $\alpha_{i}=[D /(c+1)]$, причем

$$
\sum_{i=1}^{c+1} \alpha_{i}=D
$$

Понятно, что мы всегда сможем так выбрать $\alpha_{i}, i=1,2, \ldots, c+1$. Рассмотрим величину $\Phi$, которая вычисляется для данной раскраски графа $G$ в цвета $\{1,2, \ldots, c+1\}$ следующим образом:

$$
\Phi=\sum_{i=1}^{c+1} \frac{f_{i}}{\alpha_{i}},
$$

где $f_{i}$ - число ребер графа $G$, соединяющих две вершины цвета $i, i=1,2, \ldots, c+1$. Рассмотрим такие раскраски графа $G$ в $c+1$ цвет, что значение $\Phi$ для них минимально. Обозначим множество всех таких раскрасок через $G_{c}$. Очевидно, что $G_{c}$ не пусто. Обозначим множество всех вершин цвета $i$ любой раскраски $\xi \in G_{c}$ через $\vartheta_{i}$. Тогда для любой раскраски $\xi$ графа $G$ из множества $G_{c}$ верны следующие утверждения.

Предложение 2. Для любого иุвета $i \in\{1,2, \ldots, c+1\}$ в раскраске $\xi$ и любой вершины $v \in \vartheta_{i}$, число вериин ивета $i$, смежных с $v$, не превосходит $\alpha_{i}$.

Доказательство. Предположим противное. Тогда, в силу условия

$$
\sum_{j=1}^{c+1} \alpha_{j}=D
$$

найдется такой цвет $j \neq i$, что $v$ смежна в графе $G$ менее чем с $\alpha_{j}$ вершинами цвета $j$. Перекрасив $v$ в цвет $j$, мы получим меньшее значение $Ф$. Получаем противоречие.

Предложение 3. Если какая-нибудь вершина $v \in \vartheta_{i}$ смежна в раскраске $\xi$ ровно с $\alpha_{i}$ вершинами иявета $i$, то для любого иявета $j$ вершина $v$ смежна ровно $c \alpha_{j}$ вершинами ивета $j$.

Доказательство. Предположим противное. Тогда, в силу условия

$$
\sum_{k=1}^{c+1} \alpha_{k}=D
$$

найдется такой цвет $j^{\prime} \neq i$, что $v$ смежна в графе $G$ менее чем с $\alpha_{j^{\prime}}$ вершинами цвета $j^{\prime}$, а тогда, перекрасив $v$ в цвет $j^{\prime}$, мы уменьшим значение $\Phi$. Получаем противоречие.

Предложение 4. Если в раскраске $\xi$ вершина $v \in \vartheta_{i}$ смежна хотя бы с одной вершиной своего извета, то она смежна хотя бы с одной вершиной любого другого извета.

Доказательство. В противном случае, перекрасив $v$ в цвет, с которым она не соединена, мы придем к противоречию с минимальностью $\Phi(\xi)$. 
Докажем теперь, что в множестве раскрасок $G_{c}$ есть раскраска, в которой ни для какого цвета $i$ нет клик размера $\alpha_{i}+1$ в графе $G$ на вершинах цвета $i$. Назовем такие клики большими.

Заметим, что одноцветных клик большого размера не может быть в силу предложения 2.

Для любой раскраски $\xi$ из $G_{c}$ обозначим через $\varphi(\xi)$ число больших клик в $\xi$. Обозначим через $\Omega$ множество раскрасок из $G_{c}$ с минимальным числом больших клик. Пусть $\varphi>0$ для раскрасок из $\Omega$.

Используя предложение 3 , получим следующее утверждение.

Предложение 5. Если взять вершину $v$ из какой-нибудь большой клики в какой-то раскраске $g_{c} \in \Omega$ и перекрасить ее в любой другой ијвет, то, во-первых, получится новая раскраска $g_{c}^{\prime} \in G_{c} u$, во-вторых,

$$
\varphi\left(g_{c}^{\prime}\right) \leqslant \varphi\left(g_{c}\right)
$$

Так как $\varphi$ было взято минимальным, число больших клик останется неизменным, а это значит, что обязательно появится новая большая клика в том цвете, в который мы перекрасили $v$, кроме того, мы получаем, что $g_{c}^{\prime} \in \Omega$.

Предложение 6. Пусть раскраска $\xi_{1} \in \Omega u \varphi\left(\xi_{1}\right)>0$. Пусть $C_{1}-$ большая клика иявета $i$. Рассмотрим индуциированный подграф $G_{i j}$ графа $G$ на всех вершинах иявета $i u$ ивета $j$. Тогда компонента связности в графе $G_{i j}$, содержащая $C_{1}$, представляет собой полный граф размера $\alpha_{i}+\alpha_{j}+1$.

Доказательство. Перекрасим произвольную вершину $v_{1} \in C_{1}$ в цвет $j$. Согласно предложению 5, получаем новую раскраску $\xi_{2} \in \Omega$. При этом вершина $v_{1}$ войдет в какую-то большую клику $C_{2}$ цвета $j$. Перекрасим какую-нибудь отличную от $v_{1}$ вершину $v_{2}$ клики $C_{2}$ в цвет $i$. Опять, согласно предложению 5 , получим новую раскраску $\xi_{3} \in \Omega$, в которой $v_{2}$ обязательно должна войти в какую-то большую клику $C_{3}$ цвета $i$. Продолжая таким образом, мы будем перекрашивать вершины до тех пор, пока не получим большую клику, часть вершин которой мы уже рассматривали ранее в ходе этих перекрашиваний (см. рис. 3 , где всего было сделано четыре перекрашивания и $\left.\alpha_{i}=\alpha_{j}=3\right)$. Очевидно, что если на $(k+1)$-м шаге мы получили большую клику с какими-то вершинами из $V\left(C_{l}-v_{l}\right)$, где $l \leqslant k$, то $V\left(C_{k+1}\right) \supset V\left(C_{l}-v_{l}\right)$.

Пусть мы в конце вернулись к части клики $C_{1}$, причем самих перекрашиваний было хотя бы три, то есть последняя раскраска была $\xi_{k}$, где $k \geqslant 3$. Попытаемся в изначальной раскраске $\xi_{1}$ перекрасить в клике $C_{1}$ какую-нибудь отличную от $v_{1}$ вершину $v$ в цвет $j$. По предложению 5 , получится большая клика цвета $j$. Легко видеть, что эта клика содержит все вершины $C_{k}$ кроме $v_{k-1}$, следовательно, верно следующее утверждение: любая вершина $v \in C_{1}$, где $v \neq v_{1}$, смежна со всеми вершинами в $C_{k}$ кроме вершины $v_{k-1}$, так как $v$ смежна с вершиной $v_{k}$.

Отсюда можно сделать вывод, что любая вершина $u \in C_{k}$, где $u \neq v_{k-1}$, смежна со всеми вершинами из $C_{1}$, кроме $v_{1}$.

Перекрасим вершину $v$ в раскраске $\xi_{1}$ в цвет $j$, а затем перекрасим некоторую вершину $u$ из $C_{k}$, отличную от $v_{k-1}$ и $v_{k}$, в цвет $i$ (это можно сделать, так как из определения $\alpha_{i}$ и $\alpha_{j}$ следует, что $\alpha_{i} \geqslant 2$ и $\alpha_{j} \geqslant 2$ ). Получим раскраску $\xi^{\prime} \in G_{c}$ с меньшим $\varphi$, так как $u$ не смежна с $v_{1}$ и смежна со всеми остальными вершинами из $C_{1}$. Следовательно, данный случай невозможен. Рис. 3 призван проиллюстрировать процесс перекрашивания для $k=4, \alpha_{i}=\alpha_{j}=3$. 


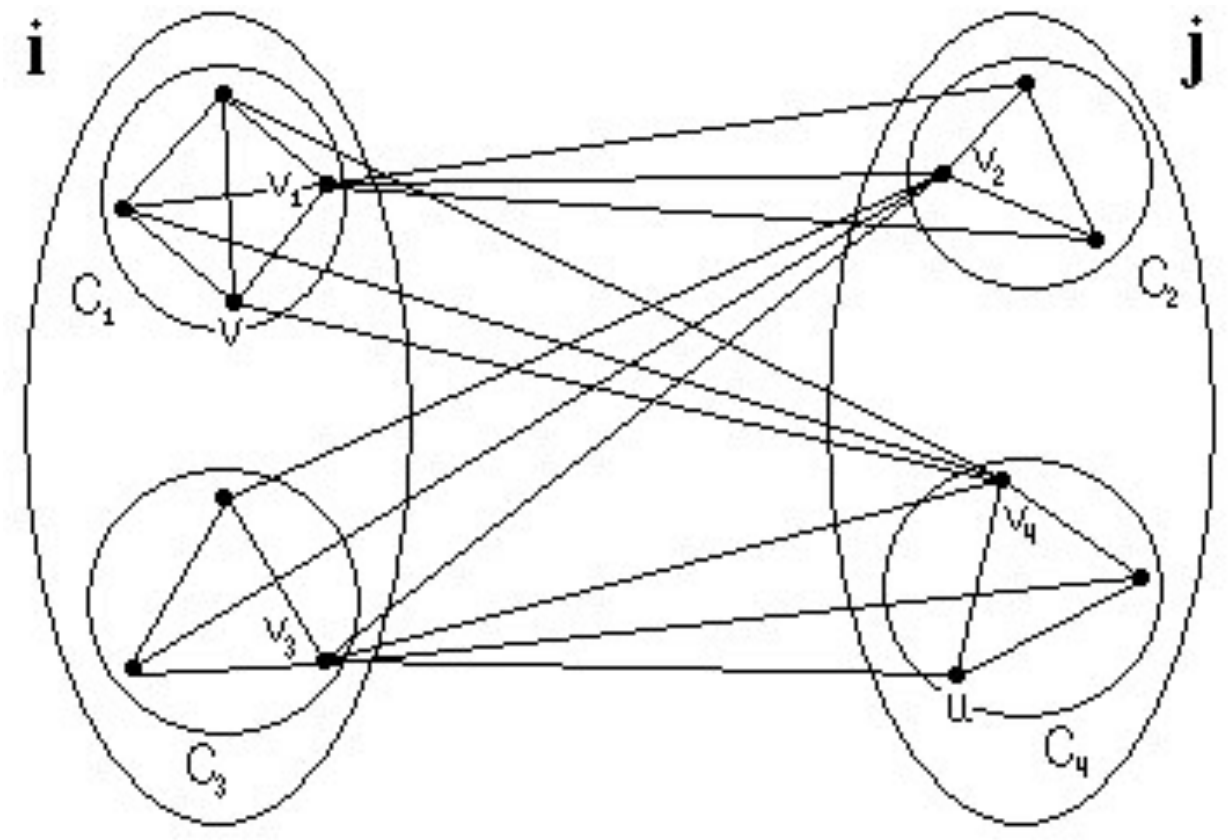

Рис. 3.

Заметим, что если мы вернулись к части клики $C_{1}$ и перекрашиваний вершин было всего два, то тогда вершина $v_{2}$ смежна со всеми вершинами из клики $C_{1}$, а значит, при перекраске любой вершины из большой клики $C_{1}$ в цвет $j$ мы получим по предложению 5 новую клику цвета $j$, содержащую $V\left(C_{2}\right) \backslash\left\{v_{1}\right\}$. Таким образом, $G\left(V\left(C_{1}\right) \cup V\left(C_{2}\right)\right)-$ полный граф. В силу произвольности выбора $v_{1}$ и $v_{2}$ и того, что граф $G\left(V\left(C_{1}\right) \cup V\left(C_{2}\right)\right)-$ клика размера $\alpha_{i}+\alpha_{j}+1$ в $G_{i j}$, мы получаем, что вершины из множества $V\left(C_{1}\right) \cup V\left(C_{2}\right)$ не смежны с другими вершинами цветов $i$ и $j$.

Если же мы вернулись в результате перекрашиваний к клике $C_{l}$, то исходя из всего выше сказанного (мы можем считать, что мы начали процесс перекрашивания с $\xi_{l}$ ) $G\left(V\left(C_{l}\right) \cup V\left(C_{l+1}\right)\right)$ образует клику в графе $G$. А тогда мы получаем $l=1$, так как вершины из множества $V\left(C_{l}\right) \cup V\left(C_{l+1}\right)$ не смежны с другими вершинами $i$-го и $j$-го цвета.

Замечание 3. Отметим, что в доказательстве предложения 6 мы существенно пользовались тем, что $\alpha_{i} \geqslant 2$ и $\alpha_{j} \geqslant 2$. Иначе мы просто не смогли бы выбрать отличную от всех $v_{i}$ вершину.

Предложение 7. В любой раскраске $g_{c} \in \Omega$ нет больщих клик.

Доказательство. Пусть у этой раскраски есть большая клика $C$, например, первого цвета. Применим предложение 6 к первому и второму цветам. Получим полный граф на вершинах 1-го и 2-го цвета размера $\alpha_{1}+\alpha_{2}+1$, содержащий $C$. Заметим, что можно произвольным образом разбить этот полный граф на две части 1-го и 2-го цвета размеров $\alpha_{1}+1$ и $\alpha_{2}$ соответственно, при этом полученная новая раскраска все равно будет лежать 
в $\Omega$. Используя предложение 6 для первого цвета и цвета $i, i \in[2, c+1]$, и предыдущее соображение, легко доказать наличие в графе $G$ полного подграфа на $1+\sum_{j=1}^{c+1} \alpha_{j}$ вершин, то есть полного подграфа размера $D+1$, что противоречит условиям теоремы 1 .

Замечание 4. На самом деле мы только что доказали теорему 3. Также отметим, что искомая в теореме 3 раскраска $\xi$ задает разбиение всех вершин графа на нужные в следствии множества.

Замечание 5. Рассмотрим конкретную раскраску $g_{c} \in \Omega$. Мы только что показали, что в раскраске $g_{c}$ нет больших клик. Таким образом, используя теорему Брукса, мы получим правильную раскраску каждого подграфа цвета $i$ в $\alpha_{i}$ цветов, в результате чего мы покрасим граф $G$ правильным образом в $D$ цветов,

$$
D=\sum_{j=1}^{c+1} \alpha_{i} .
$$

Если какая-то вершина в раскраске $g_{c}$ была смежна с вершиной своего цвета, то согласно предложению 4 у нее в окрестности (уже в раскраске в $D$ цветов) будет хотя бы $c+1$ различных цветов, то есть основная проблема заключается в раскраске “вырожденных" вершин, то есть вершин, несмежных со своим и еще каким-нибудь цветом, раскраски $g_{c}$. Действительно, если бы $G$ был изначально двудольным, теорема о его раскраске в $D$ цветов с выполнением условия про большое число различных цветов в окрестности каждой вершины была бы совсем неочевидной. На самом деле доказательство этой теоремы для случая двудольного графа весьма наглядно показывает, в чем собственно состоит сложность и специфика данной задачи, поэтому этот случай полезно иметь в виду.

Рассмотрим раскраску $g_{c} \in \Omega$ и в ней все вершины, которые смежны с вершинами не более чем $c-1$ различных цветов. Обозначим $\Upsilon$ множество всех таких вершин. Заметим, что любая вершина $v \in \Upsilon$, во-первых, не смежна ни с одной вершиной своего цвета в раскраске $g_{c}$ и, во-вторых, есть еще один отличный от цвета $v$ цвет такой, что $v$ не смежна ни с одной вершиной этого цвета. Таким образом, эту вершину $v \in \Upsilon$ всегда можно перекрасить в другой цвет так, чтобы полученная новая раскраска по-прежнему была из $\Omega$. Более того, можно перекрасить все или только часть вершин из $\Upsilon$ некоторого цвета $i$ так, чтобы полученная раскраска по-прежнему была из $\Omega$ (естественно, перекрашивать мы их будем скорее всего в различные цвета).

Для любой вершины $v \in \Upsilon$ найдется такой цвет в раскраске $g_{c}$, что $v$ соединена по крайней мере $\mathrm{c}\lceil p /(c-1)\rceil$ вершинами этого цвета. Разобьем $\Upsilon$ на $c+1$ множество $\theta_{1}, \theta_{2}, \ldots, \theta_{c+1}$ таким образом, что любая вершина из $\theta_{i}$ смежна по крайней мере с $\lceil p /(c-1)\rceil$ вершинами именно цвета $i$.

Для всех $i \in[1, c+1]$ обозначим через $H_{i}$ индуцированный подграф графа $G$ на множестве вершин цвета $i$ в раскраске $g_{c}$.

Предложение 8. Для любой вершиньл $v \in H_{i}$

$$
\left\lceil d_{H_{i}}(v)+\frac{d_{G\left(\theta_{i} \cup\{v\}\right)}(v)}{c+2}\right\rceil \leqslant \alpha_{i} .
$$

Доказательство. Рассмотрим множество $E_{v}$ всех ребер в графе $G$ с одним концом в вершине $v$. Очевидно, что $\left|E_{v}\right| \leqslant D$. Рассмотрим множество $E_{1} \subseteq E_{v}$ всех ребер из $E_{v}$, у которых смежная с $v$ вершина не лежит в $\theta_{i}$. Пусть из $v$ ведет в некоторый цвет $j$, отличный от $i$, меньше, чем $\alpha_{j} d_{H_{i}}(v) / \alpha_{i}$ ребер из множества $E_{1}$. Тогда перекрасим все 


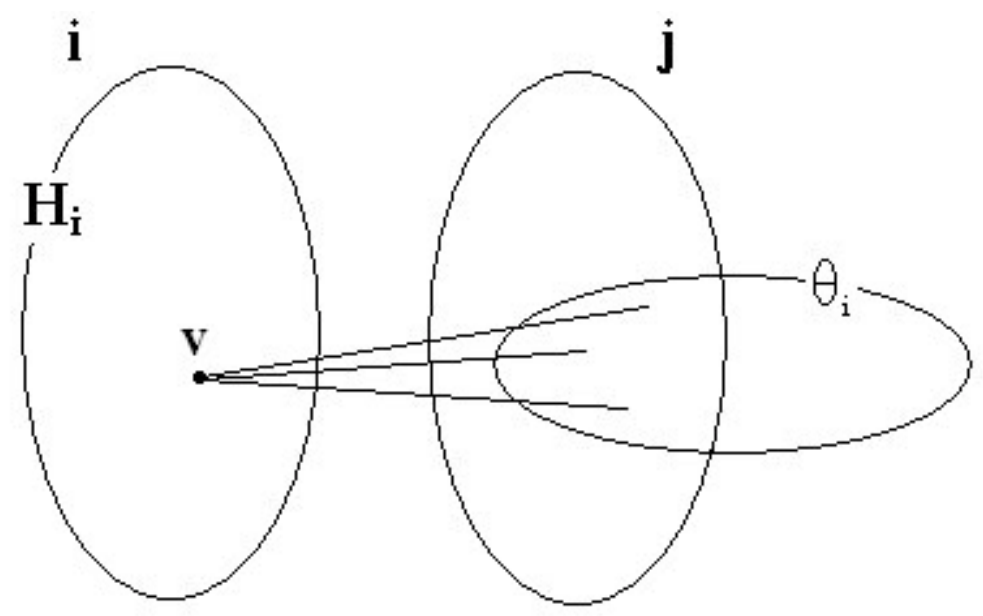

Рис. 4.

вершины цвета $j$ множества $\theta_{i} \subseteq \Upsilon$ так, чтобы полученная раскраска по-прежнему была из $\Omega$. Очевидно, мы перекрасим эти вершины не в цвет $i$, поэтому величина $d_{H_{i}}(v)$ в новой раскраске не изменит своего значения. Перекрасим в новой раскраске $v$ в цвет $j$. Заметим, что к значению Ф для полученной раскраски добавится величина, меньшая чем

$$
\frac{\left(\alpha_{j} / \alpha_{i}\right) d_{H_{i}}(v)}{\alpha_{j}}-\frac{d_{H_{i}}(v)}{\alpha_{i}}=0,
$$

таким образом, мы нашли раскраску с меньшим значением $\Phi$ и получили противоречие.

Тогда получаем, что всего из вершины $v$ в графе $G$ ведет по крайней мере $R$ ребер, где

$$
\begin{aligned}
\left|E_{v}\right| & \geqslant d_{H_{i}}(v)+d_{G\left(\theta_{i} \cup\{v\}\right)}(v)+\sum_{j \neq i} \frac{\alpha_{j}}{\alpha_{i}} d_{H_{i}}(v) \\
& =\sum_{j=1}^{c+1} \frac{\alpha_{j}}{\alpha_{i}} d_{H_{i}}(v)+d_{G\left(\theta_{i} \cup\{v\}\right)}(v) \\
& =\frac{D}{\alpha_{i}} d_{H_{i}}(v)+d_{G\left(\theta_{i} \cup\{v\}\right)}(v) .
\end{aligned}
$$

По определению,

$$
R \leqslant\left|E_{v}\right| \leqslant D .
$$

Получаем, что

$$
D \geqslant \frac{D}{\alpha_{i}} d_{H_{i}}(v)+d_{G\left(\theta_{i} \cup\{v\}\right)}(v) \Longrightarrow \alpha_{i} \geqslant d_{H_{i}}(v)+\frac{\alpha_{i}}{D} d_{G\left(\theta_{i} \cup\{v\}\right)}(v) .
$$


Используя то, что

$$
\begin{aligned}
\alpha_{i} & \geqslant\left[\frac{D}{c+1}\right], \\
D & \geqslant\left(c^{3}+8 c^{2}+19 c+6\right)(c-1),
\end{aligned}
$$

получим неравенство

$$
\frac{\alpha_{i}}{D}>\frac{1}{c+2}
$$

Тогда

$$
\alpha_{i} \geqslant d_{H_{i}}(v)+\frac{d_{G\left(\theta_{i} \cup\{v\}\right)}(v)}{c+2} .
$$

\section{4. Вторая часть доказательства теоремы 1}

Лемма 1. Пусть дано два непустых множества вериин $A$ и $B$ и некоторый связный граф $H=(A \cup B, E)$, обозначим через $\mathscr{G}$ индуцированный подграф $H(B)$. Определим $d_{A}(v)$, где $v \in B$, как число ребер, ведущих из вершины $v$ в множество А. Пусть граф $H$ обладает следующими свойствами:

(1) никакие две вериины множества А не соединены ребром;

(2) степень каждой вершины из А в графе $H$ не меньше $q^{3}+2 q^{2}-q-8$, где $q \geqslant 4$;

(3) для любой вершины $v \in B$ выполняется неравенство

$$
d_{\varphi}(v)+\left\lceil\frac{d_{A}(v)}{q}\right\rceil \leqslant d,
$$

где

$$
d \geqslant q^{3}+2 q^{2}-q-8
$$

Тогда можно раскрасить граф $\mathscr{G}$ правильным образом в $d$ иветов так, чтобы для любой вершины $v \in A$ среди ее соседей в $B$ были вершины по крайней мере q различных ияветов.

Замечание 6. Заметим, что

$$
(c+2)^{3}+2(c+2)^{2}-(c+2)-8=c^{3}+8 c^{2}+19 c+6 .
$$

Замечание 7. В лемме 1 множество вершин $B$ обозначает то, что в первой части доказательства обозначалось $H_{i}$, множество вершин $A$ обозначает то, что раньше обозначалось $\theta_{i}$, причем при этом неважно, были ли между вершинами из $\theta_{i}$ какие-нибудь ребра. Нам важно только, с какими вершинами из $H_{i}$ были смежны вершины $\theta_{i}$, ибо красить мы будем только вершины из $H_{i}$.

Через $q$ мы обозначим в лемме 1 то, что в первой части доказательства теоремы было равно $c+2$, а через $d$ обозначим то, чему раньше было равно $\alpha_{i}$. Через $H$ в лемме 1 мы обозначим граф $G\left(\theta_{i} \cup H_{i}\right)-E\left(G\left(\theta_{i}\right)\right)$. По определению множества $\theta_{i}$, из любой вершины $v \in \theta_{i}$ вело по крайней мере $p /(c-1)>q^{3}+2 q^{2}-q-8$ ребер в множество вершин $V\left(H_{i}\right)$. 
Мы будем полагать в лемме 1, что граф $H$ связен (можно все доказать для компонент связности). Кроме этого можно считать, что множество $\theta_{i}$ непусто, иначе мы получаем обычную теорему Брукса, так как тогда нам нужно всего лишь покрасить правильным образом граф $H_{i}$ в $\alpha_{i}$ цветов, а мы знаем, что в графе $H_{i}$ нет полных подграфов на $\alpha_{i}+1$ вершине (в графе $H_{i}$ нет больших клик) и что

$$
d_{H_{i}}(v)=\left\lceil d_{H_{i}}(v)+\frac{d_{G\left(\theta_{i} \cup\{v\}\right)}(v)}{c+2}\right\rceil \leqslant \alpha_{i} .
$$

Таким образом, все условия леммы 1 выполнены для множеств $B=V\left(H_{i}\right)$ и $A=\theta_{i}$.

Если мы, используя лемму 1 для множеств $B=V\left(H_{i}\right)$ и $A=\theta_{i}$, каждый подграф $H_{i}$ графа $G$ в раскраске $g_{c}$ раскрасим правильным образом в $\alpha_{i}$ новых цветов так, что каждая вершина из $\theta_{i}$ будет смежна с вершинами по крайней мере $c$ различных цветов в графе $H_{i}$, то мы получим правильную раскраску в $D$ цветов всего графа $G$, при этом, как легко заметить, все вершины из множества

$$
\Upsilon=\bigcup_{i=1}^{c+1} \theta_{i}
$$

будут смежны с вершинами по крайней мере $c$ различных цветов. Кроме того в силу определения множества $\Upsilon$ все вершины из множества $V(G) \backslash \Upsilon$ будут смежны по крайней мере с $c$ различными группами цветов, а значит, и просто с $c$ вершинами различных цветов. Таким образом, мы свели теорему 1 к лемме 1 и нам осталось только доказать лемму 1 .

Замечание 8. Вся вторая часть будет посвящена доказательству леммы 1. Поэтому, дабы избежать непонимания, связанного с совпадением обозначений, сразу оговорим то, что обозначения в первой и второй частях не имеют никакой связи, то есть эти части автономны, и не следует искать определения какой-нибудь величины из второй части доказательства в первой части.

Замечание 9. В утверждении леммы 1 можно заменить $q$ на $q-2$, но для удобства дальнейших выкладок мы этого делать не будем.

Доказательство леммы 1. Предположим, что лемма 1 неверна, тогда рассмотрим минимальный по числу вершин граф $H$, удовлетворяющий всем условиям леммы 1 , для которого неверно утверждение леммы 1.

Назовем допустимым множество $S_{i} \subseteq B$, если $S_{i} \subset N_{H}\left(v_{i}\right)$, где $v_{i} \in A$ и $\left|S_{i}\right|=q$. Обозначим через $\Lambda$ множество всевозможных таких выборок допустимых множеств $S_{i}$ для всех $i \in\{1,2, \ldots,|A|\}$.

Утверждение леммы вытекает из следующего факта: для каждой вершины $v_{i}$ из $A$ можно выбрать допустимое множество $S_{i}$ таким образом, что если добавить к ребрам $E(\mathscr{G})$ все полные графы на множествах $S_{i}$, то вершины полученного нового графа $\widetilde{\mathscr{G}}$ можно раскрасить в $d$ цветов правильным образом.

Будем рассматривать $\widetilde{\mathscr{G}}$ как граф с кратными ребрами. Таким образом, мы получили равносильную переформулировку леммы 1.

Для доказательства раскрашиваемости $\widetilde{\mathscr{G}}$ в $d$ цветов оказывается удобным проводить некоторую редукцию графа, а именно, пусть в графе $\widetilde{\mathscr{G}}$ есть некоторая вершина $\hat{v}$ степени, меньшей $d$, тогда можно выкинуть эту вершину из графа $\widetilde{\mathscr{G}}$ и проводить доказательство для графа $\widetilde{\mathscr{G}} \backslash \hat{v}$. 
Будем говорить, что $\hat{v}$ рекурсивно удаляется из графа $\widetilde{\mathscr{G}}$, если можно провести последовательность таких редукций, чтобы последней в этой последовательности редуцировалась вершина $\hat{v}$. Граф $\widetilde{G}$ будем называть рекурсивным, если он последовательно редуцируется до пустого графа. Такую редукцию мы назвали рекурсией, так как если граф редуцируется до пустого, то красить его можно, начиная с последней удаленной вершины и кончая первой, то есть процесс раскраски представляет собой обычную рекурсию.

На самом деле мы будем доказывать более сильный факт, именно, вместо утверждения о том, что $\widetilde{\mathscr{G}}$ красится правильным образом в $d$ цветов, мы будем доказывать, что $\widetilde{\mathscr{G}}$ является рекурсивным относительно раскраски в $d$ цветов.

Обозначим через $S$ множество тех вершин из $B$, которые смежны хотя бы с одной вершиной из $A$. Докажем, что для графа $H$ выполнена усиленная переформулировка факта в предположении, что $H$ - минимальный по числу вершин граф, для которого не верна лемма 1. Таким образом мы получим противоречие, а значит, докажем лемму 1.

Определение 3. Определим для каждой вершины $v$ из множества $B$ величину $L(v)$, полагая

$$
L(v)=d_{\varphi}(v)+\frac{d_{A}(v)}{q+1} .
$$

Замечание 10. Заметим, что если выбрать $S_{i}$ случайным образом (независимо для каждой вершины $v_{i}$, причем так, что для каждой вершины $v_{i}$ все возможные варианты множества $S_{i}$ равновероятны), то математическое ожидание степени каждой вершины $v$ из

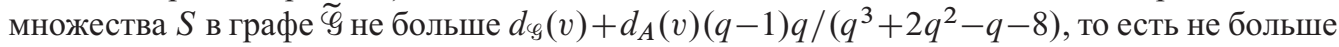
$L(v)$ (заметим, что так как $q \geqslant 4$, справедливо неравенство $q^{3}+2 q^{2}-q-8>q\left(q^{2}-1\right)$ ), а значит, по третьему условию леммы 1 меньше $d$. Таким образом, в среднем степень каждой вершины в $\widetilde{\mathscr{G}}$ меньше $d$. Это дает нам надежду на то, что граф $\widetilde{\mathscr{G}}$ окажется рекурсивным, то есть, если мы последовательно будем удалять из $\widetilde{G}$ вершины степени, меньшей, чем $d$, то в конце концов получим пустой граф.

Для завершения доказательства леммы нам осталось соответствующим образом выбрать $S_{i}$ (так, чтобы $\widetilde{G}$ стал рекурсивным).

Определение 4. Сдвигом нескольких допустимых множеств $S_{i_{1}}, S_{i_{2}}, \ldots, S_{i_{z}}$ в выборке $\lambda \in \Lambda$ назовем замену $\lambda$ на $\lambda^{\prime} \in \Lambda$, где $\lambda^{\prime}$ отличается от $\lambda$ только тем, что все допустимые множества $S_{i_{1}}, S_{i_{2}}, \ldots, S_{i_{z}}$ заменены в ней на некоторые другие допустимые множества $S_{i_{1}}^{\prime}, S_{i_{2}}^{\prime}, \ldots, S_{i_{z}}^{\prime}$. Множества $S_{i_{1}}^{\prime}, S_{i_{2}}^{\prime}, \ldots, S_{i_{z}}^{\prime}$ будем называть результатом сдвига множеств $S_{i_{1}}, S_{i_{2}}, \ldots, S_{i_{z}}$.

Обозначим через $R$ множество $B \backslash S$. Можно считать, что степень любой вершины из $R$ равна $d$, так как по условию леммы 1 степень любой вершины из $B$ в графе $\mathscr{G}$ меньше либо равна $d$, а если степень вершины меньше $d$, то можно сразу выкинуть рекурсивно эту вершины из $\widetilde{\mathscr{G}}$, для любой допустимой выборки.

Предложение 9. Пусть $F-$ такой граф, что $V(F)=F_{1} \cup F_{2} u F_{1} \cap F_{2}=\varnothing$. Степень любой вершины из $F_{2}$ в графе $F$ меньше либо равна $D$, кроме того в $F$ существует такая вершина $v \in F_{1}$, что граф $F\left(F_{2} \cup\{v\}\right)$ связен, $d_{F}(v)<D$ и вершина $v$ смежна со всеми остальныли вершинами в $F_{1}$. Пусть граф $F\left(F_{1}\right)$ уже раскрашен правильньм образом 6 $D$ ияветов. Тогда все вершины графа $F$ можно докрасить до правильной раскраски в $D$ ияветов.

Доказательство. Выкинем из графа $F$ вершину $v$, получится новый граф $F^{\prime}$. На множестве $F_{1} \backslash\{v\}$ уже есть правильная раскраска в $D$ цветов. По одной вершине рекурсивно 

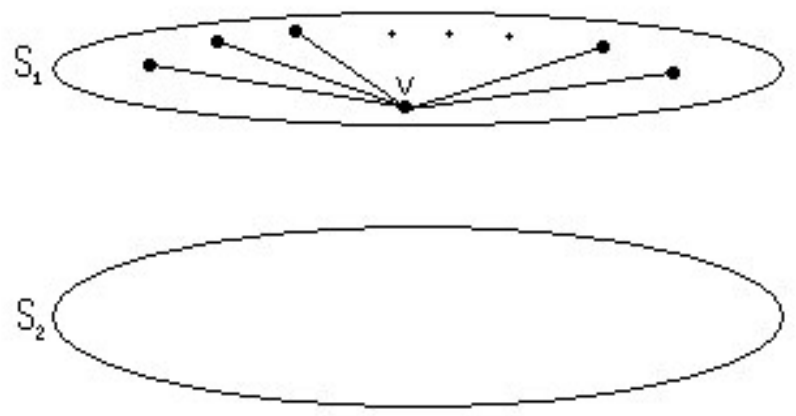

Рис. 5.

докрасим правильным образом все вершины из $F_{2}$, так можно сделать в силу связности графа $F\left(F_{2} \cup\{v\}\right)$ и условия, что степень в графе $F$ любой вершины из $F_{2}$ меньше либо равна $D$. Перенесем полученную правильную раскраску графа $F^{\prime}$ в $D$ цветов на $F$ и покрасим $v$ в какой-нибудь цвет, отличный от всех цветов вершин из $N_{F}(v)$ (так можно сделать, так как по условию $d_{F}(v)<D$ ). В итоге мы покрасили правильным образом в $D$ цветов граф $F$, правда при этом мы, возможно, изменили цвет вершины $v$ по сравнению с заданной раскраской на $F_{1}$. Заметим, что все вершины из множества $F_{1} \backslash\{v\}$ будут окрашены в цвета, отличные от цвета $v$ в исходной раскраске $F_{1}$, так как эта раскраска была правильной для графа $F\left(F_{1}\right)$, а вершина $v$ смежна со всеми остальными вершинами в $F_{1}$, кроме того заметим, что все цвета вершин из множества $F_{1} \backslash\{v\}$ отличны от цвета $v$ в раскраске $F$. Теперь, если мы изменили цвет вершины $v$ по сравнению с исходной раскраской $F_{1}$, то просто поменяем цвет, совпадающий с цветом $v$ в исходной раскраске $F_{1}$, и нынешний цвет $v$ в раскраске $F$ местами. Получится опять правильная раскраска $F$, но уже полностью совпадающая с исходной раскраской на множестве $F_{1}$.

Определение 5. Регулярным сдвигом множеств $S_{i}$, некоторого набора $v \in \Lambda$ относительно множества $S^{\prime}$ мы будем называть такой сдвиг множеств $S_{i}$, где $i \in\{1, \ldots,|A|\}$, в множества $S_{i}^{\prime}, i \in\{1, \ldots,|A|\}$, что для всех $i \in\{1, \ldots,|A|\}$ множество $S_{i} \cap S^{\prime}$ содержит множество $S_{i}^{\prime} \cap S^{\prime}$. Если найдется такое $i \in\{1, \ldots,|A|\}$, что $\left|S_{i} \cap S^{\prime}\right|$ больше $\left|S_{i}^{\prime} \cap S^{\prime}\right|$, то такой регулярный сдвиг мы будем называть невырожденным.

Замечание 11. Регулярный сдвиг относительно некоторого множества есть регулярный сдвиг относительно любого подмножества этого множества, правда невырожденность при этом не обязательно сохраняется.

Предложение 10. Пусть

$$
\eta=\left\{S_{1}, S_{2}, \ldots, S_{|A|}\right\}
$$

- набор допустимых множеств графа $H$, являющцегося минимальным по числу вершин контрпримером к лемме 1 , и пусть $S^{\prime} \subseteq S, R^{\prime} \subseteq R-$ такие множества, что все вершинь 


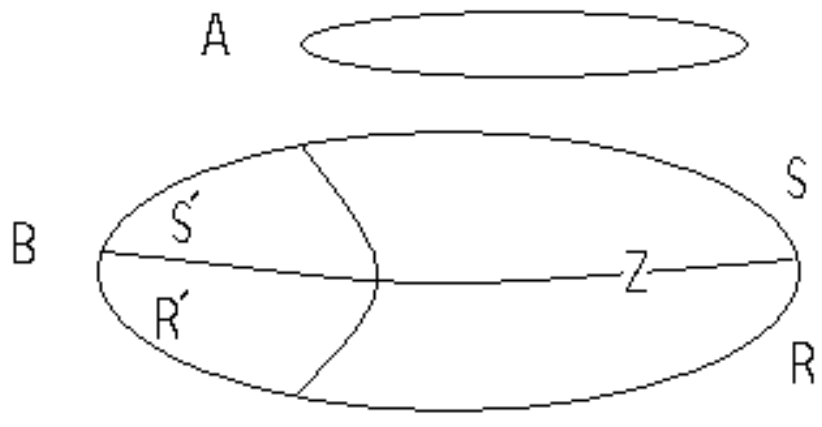

Pис. 6.

из $B \backslash\left(S^{\prime} \cup R^{\prime}\right)$ рекурсивно удаляются из графа $\widetilde{\mathscr{G}}$, причем

$$
\begin{aligned}
d_{\widetilde{G}}(u) & =d, & & u \in R, \\
d_{\widetilde{\mathscr{G}}\left(S^{\prime} \cup R^{\prime}\right)}(u) & =d, & & u \in R^{\prime} .
\end{aligned}
$$

Кроме того, пусть

$$
\tilde{H}^{\prime}=\widetilde{\mathscr{G}}\left(S^{\prime} \cup R^{\prime}\right)
$$

$u$

$$
\sum_{u \in \tilde{H}^{\prime}} d_{\tilde{H}^{\prime}}(u)>\sum_{u \in \tilde{H}^{\prime}} L(u) .
$$

Тогда можно регулярно сдвинуть невырожденным образом несколько множеств $S_{i}$ относительно множества $S^{\prime} \cup R^{\prime}$ так, чтобы $B \backslash\left(S^{\prime} \cup R^{\prime}\right)$ по-прежнему рекурсивно удалялось из полученного в результате этого сдвига графа $\widetilde{\mathscr{G}}^{\prime}$.

Доказательство. Доказывать это утверждение будем индукцией по размеру множества $B \backslash\left(S^{\prime} \cup R^{\prime}\right)$.

Что касается основания индукции, то случай $\left|B \backslash\left(S^{\prime} \cup R^{\prime}\right)\right|=0$, очевидно, невозможен, так как в силу замечания 10 не будет выполнено условие (2).

Докажем возможность перехода от всех меньших, чем $k$, размеров множества $B \backslash\left(S^{\prime} \cup R^{\prime}\right)$ к размеру $k$.

Пусть $Z=B \backslash\left(S^{\prime} \cup R^{\prime}\right)$ (см. рис. 6).

Пусть есть множества $S^{\prime}$ и $R^{\prime}$ такие, что $|Z|=k$ и для них не выполнено утверждение индукционного перехода.

Покажем, что существует такая вершина $v_{i} \in A$ и соответствующее ей множество $S_{i}$, что $S_{i}$ можно регулярно сдвинуть относительно $S^{\prime}$ невырожденным образом. Предположим противное, тогда для любого допустимого множества $S_{i}$ и соответствующей ему вершины $v_{j} \in A$ допускается всего две возможности: 


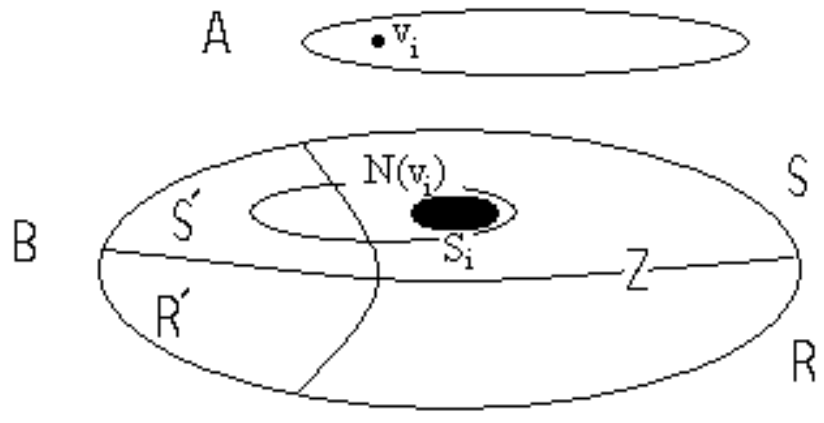

Рис. 7.

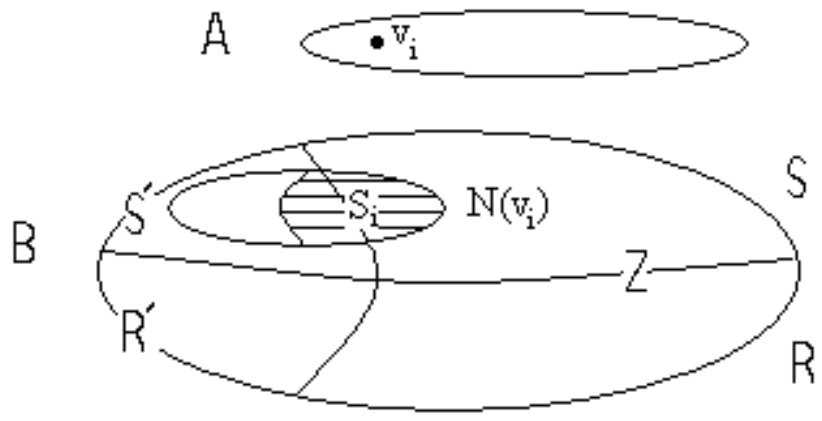

Рис. 8.

(1) множество $S_{i} \cap S^{\prime}=\varnothing$ (см. рис. 7);

(2) множество $N_{H}\left(v_{i}\right) \backslash S^{\prime} \subseteq S_{i}$ (см. рис. 8).

Как нетрудно заметить, число добавленных ребер с концами в множестве вершин $S^{\prime}$ в обоих этих случаях достигает своего минимального значения. Таким образом, верна 
цепочка неравенств

$$
\begin{aligned}
\sum_{v \in V\left(\tilde{H}^{\prime}\right)} d_{\tilde{H}^{\prime}}(v) & \leqslant \sum_{v \in V\left(\tilde{H}^{\prime}\right)} E\left(d_{\widetilde{H}^{\prime}}(v)\right) \\
& \leqslant \sum_{v \in V\left(\tilde{H}^{\prime}\right)} E\left(d_{\widetilde{g}}(v)\right) \leqslant \sum_{v \in V\left(\tilde{H}^{\prime}\right)} L(v),
\end{aligned}
$$

где $E(\cdot)$ обозначает математическое ожидание случайной величины (с оговоренным в замечании 10 распределением). По условию (2)

$$
\sum_{u \in \widetilde{H}^{\prime}} d_{\widetilde{H}^{\prime}}(u)>\sum_{u \in \widetilde{H}^{\prime}} L(u) .
$$

Получаем противоречие с предыдущим неравенством.

Значит, существует такая вершина $v_{i} \in A$, что часть ее окрестности, находящаяся в $Z$, не лежит в соответствующем множестве $S_{i}$, а множество $S_{i} \cap S^{\prime} \neq \varnothing$.

Таким образом, можно рассмотреть такую вершину $v \in N_{H}\left(v_{i}\right)$, что она не лежит ни в множестве $S^{\prime}$, ни в множестве $S_{i}$, при этом часть множества $S_{i}$ лежит в множестве $S^{\prime}$. Известно, что $Z$ рекурсивно удаляется из $\widetilde{G}$. Начнем удалять рекурсивно вершины из $Z$, причем будем рекурсивно удалять, пока это возможно, вершины отличные от $v$. В какой-то момент мы не сможем ничего удалить, кроме $v$. Это значит, что от множества $Z$ остались вершины $v, u_{1}, u_{2}, \ldots, u_{l} \in S, w_{1}, w_{2}, \ldots, w_{m} \in R$.

Обозначим множество всех оставшихся в $Z$ вершин через $P$, а через $\tilde{I}$ обозначим индуцированный граф $\widetilde{\mathscr{G}}\left(S^{\prime} \cup R^{\prime} \cup P\right)$.

Заметим, что в графе $\tilde{I}$ степени всех вершин $u_{k}$, где $k \in[1, l]$, и всех вершин $w_{j}$, где $j \in[1, m]$, не меньше $d$. Заметим также, что степень вершины $v$ в $\tilde{I}$ меньше $d$.

Если степень вершины $v$ меньше $d-q+1$, то сдвинем $S_{i}$ в множество $S_{i}^{\prime}$ следующим образом: возьмем вершину $x$ из $S_{i}$, которая лежит в множестве $S^{\prime} \cap S_{i}$ (такая вершина $x$ обязательно найдется, так как $\left.S^{\prime} \cap S_{i} \neq \varnothing\right)$, тогда положим

$$
S_{i}^{\prime}=\left(S_{i} \backslash\{x\}\right) \cup\{v\} .
$$

Остальные множества из набора $\eta$ двигать не будем. Заметим, что описанный нами сдвиг будет регулярным и невырожденным относительно множества $S^{\prime}$, также понятно, что множество $Z$ по-прежнему можно рекурсивно удалить (очевидно, что можно так же, как и раньше, рекурсивно удалить все вершины из $Z \backslash P$, потом можно рекурсивно удалить вершину $v$, так как степень вершины $v$ в графе $\tilde{I}$ была меньше $d-q+1$, а значит, после сдвига стала не больше $d-1$, а потом можно рекурсивно удалить оставшиеся в $Z$ вершины, так как множество $Z$ рекурсивно удалялось из графа $\widetilde{\mathscr{G}}$ и новых ребер в графе $\tilde{I}-\{v\}$ при описанном выше сдвиге не добавилось). В этом случае мы доказали индукционный переход.

Предположим противное, пусть степень $v$ меньше $d$, но не меньше $d-q+1$.

Докажем, что уже для графа $\tilde{I}$ выполнено неравенство

$$
\sum_{u \in \tilde{I}} d_{\widetilde{I}}(u)>\sum_{u \in \tilde{I}} L(u) .
$$

Пусть это утверждение доказано, тогда можно применить предположение индукции для множеств $S_{0}^{\prime}$ и $R_{0}^{\prime}$, где

$$
\begin{aligned}
& S_{0}^{\prime}=\left(S^{\prime} \cup R^{\prime} \cup P\right) \cap S, \\
& R_{0}^{\prime}=\left(S^{\prime} \cup R^{\prime} \cup P\right) \cap R,
\end{aligned}
$$




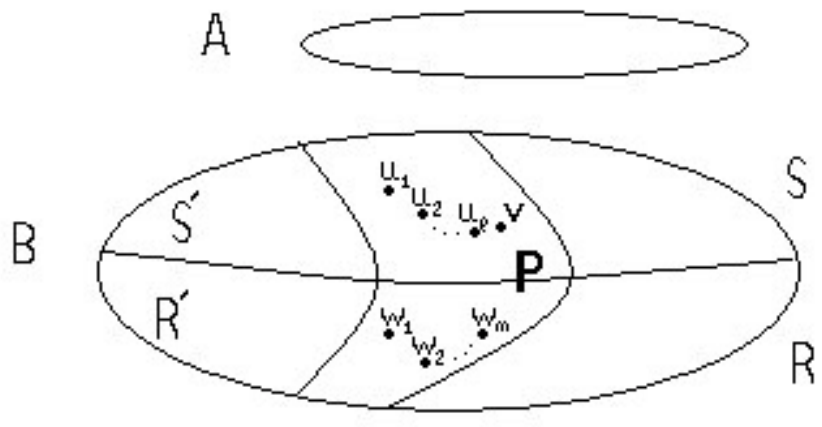

Рис. 9.

то есть мы можем регулярно сдвинуть невырожденным образом набор множеств $\eta$ относительно $S^{\prime} \cup R^{\prime} \cup P$ так, чтобы вершины из $B \backslash\left(S^{\prime} \cup R^{\prime} \cup P\right)$ по-прежнему удалялись рекурсивно в полученном графе. Заметим, что если набор сдвинулся регулярно относительно $S^{\prime} \cup R^{\prime} \cup P$, то он сдвинулся регулярно относительно $S^{\prime} \cup R^{\prime}$. Кроме того, заметим, что в полученном в итоге сдвига графе будут по-прежнему рекурсивно удаляться все вершины из множества $B \backslash\left(S^{\prime} \cup R^{\prime}\right)$, так как вначале можно рекурсивно удалить все вершины множества $B \backslash\left(S^{\prime} \cup R^{\prime} \cup P\right)$, а затем удалить рекурсивно все вершины из $P$, поскольку новых ребер в графе $\tilde{I}$ в результате такого сдвига не добавилось.

Будем продолжать до тех пор пока одно из множеств $S_{i}$ регулярно не сдвинется невырожденным образом относительно $S^{\prime} \cup R^{\prime}$, либо пока степень вершины $v$ не станет меньше $d-q+1$, либо степень какой-то вершины из $P \backslash\{v\}$ не станет меньше $d$. В последнем случае мы рекурсивно сможем еще выкинуть несколько вершин из $Z$ и уже для нового меньшего графа $\tilde{I}$ применить те же самые рассуждения. Здесь необходимо отметить, что рано или поздно мы обязательно придем к одному из этих вариантов, так как иначе мы будем бесконечное число раз делать регулярный невырожденный сдвиг относительно множества $S^{\prime} \cup R^{\prime} \cup P$, а значит, сколь угодно долго уменьшать значение суммы $\sum_{i=1}^{|A|}\left|S_{i} \cap\left(S^{\prime} \cup R^{\prime} \cup P\right)\right|$.

Обозначим через $l^{\prime}$ число ребер, ведущих из множества вершин $P$ в множество вершин $S^{\prime}$.

В силу условий предложения 10 , состоящих в том, что $d \widetilde{q}_{\left(S^{\prime} \cup R^{\prime}\right)}(u)=d$ для всех $u \in R^{\prime}$ и $d_{\widetilde{g}}(u)=d$ для всех $u \in R$, не может быть ребер между множествами $P$ и $R^{\prime}$.

Для завершения доказательства предложения 10 осталось доказать, что для графа $\tilde{I}=\widetilde{\mathscr{G}}\left(S^{\prime} \cup R^{\prime} \cup P\right)$ выполнено неравенство (3). Предположим противное. Тогда

$$
\begin{aligned}
\sum_{u \in \tilde{I}} L(u) & \geqslant \sum_{u \in \tilde{I}} d_{\tilde{I}}(u) \geqslant \sum_{u \in \tilde{H}^{\prime}} d_{\tilde{H}^{\prime}}(u)+l^{\prime}+\sum_{u \in P} d_{\tilde{I}}(u) \\
& >\sum_{u \in \tilde{H}^{\prime}} L(u)+\sum_{u \in P} d_{\tilde{I}^{\prime}}(u)+l^{\prime}
\end{aligned}
$$


Таким образом, мы получаем, что

$$
\sum_{u \in P} L(u)>l^{\prime}+\sum_{u \in P} d_{\tilde{I}}(u),
$$

а значит,

$$
l^{\prime}+\sum_{u \in P}\left(d_{\widetilde{I}}(u)-L(u)\right)<0 .
$$

Оценим величину $d_{\tilde{I}}\left(u_{i}\right)-L\left(u_{i}\right)$ для всех $i \in\{1, \ldots, l\}$. По определению $L(u)$ и в силу того, что $d_{\tilde{I}}\left(u_{i}\right) \geqslant d$ получаем, что для любого $i \in[1, l]$ верно неравенство

$$
d_{\widetilde{I}}\left(u_{i}\right)-L\left(u_{i}\right) \geqslant d-d_{\varphi}\left(u_{i}\right)-\frac{d_{A}\left(u_{i}\right)}{q+1} .
$$

Используя неравенство (1), получим, что

$$
\begin{aligned}
d_{\tilde{I}}\left(u_{i}\right)-L\left(u_{i}\right) & \geqslant d-d_{\varphi}\left(u_{i}\right)-\frac{d_{A}\left(u_{i}\right)}{q+1} \\
& \geqslant d_{\varphi}\left(u_{i}\right)+\left\lceil\frac{d_{A}\left(u_{i}\right)}{q}\right\rceil-d_{\varphi}\left(u_{i}\right)-\frac{d_{A}\left(u_{i}\right)}{q+1} .
\end{aligned}
$$

Таким образом,

$$
d_{\tilde{I}}\left(u_{i}\right)-L\left(u_{i}\right) \geqslant\left\lceil\frac{d_{A}\left(u_{i}\right)}{q}\right\rceil-\frac{d_{A}\left(u_{i}\right)}{q+1} .
$$

Далее, $d_{A}\left(u_{i}\right)>0$ для всех $i \in[1, l]$, так как $u_{i} \in S$ для всех $i \in\{1, \ldots, l\}$. Рассмотрим следующие случаи:

(a) $0<d_{A}\left(u_{i}\right) \leqslant q$;

(б) $d_{A}\left(u_{i}\right) \geqslant q+1$.

В обоих случаях выполнено неравенство

$$
d_{\widetilde{I}}\left(u_{i}\right)-L\left(u_{i}\right) \geqslant\left\lceil\frac{d_{A}\left(u_{i}\right)}{q}\right\rceil-\frac{d_{A}\left(u_{i}\right)}{q+1} \geqslant \frac{1}{q+1} .
$$

Пусть

$$
q_{1}=d_{\tilde{I}}(v)-d+q,
$$

тогда, как мы показали выше, $q_{1}>0$. Заметим, что для вершины $v$, аналогично выкладкам (3) и (6), можно получить неравенство

$$
d_{\tilde{I}}(v)-L(v) \geqslant q_{1}-q+\frac{1}{q+1} .
$$

Справедливо включение $w_{i} \in R$, где $i \in\{1, \ldots, m\}$, поэтому $d \varphi\left(w_{i}\right)=L\left(w_{i}\right)$, кроме того, вершины из множества $P \cap R$ нельзя рекурсивно удалить из графа $\tilde{I}$. Используя еще условие предложения 10 , состоящее в том, что $d g(u)=d$ для любой вершины $u \in R$, получаем равенство

$$
d_{\tilde{I}}\left(w_{j}\right)=d_{\varphi}\left(w_{j}\right)=d
$$


Тогда для всех $i \in\{1, \ldots, m\}$ верно равенство

$$
d=d_{\widetilde{I}}\left(w_{i}\right)=L\left(w_{i}\right) .
$$

Подставляя теперь неравенства (6), (7) и равенство (8) в неравенство (3), получим неравенство

$$
l^{\prime}+l \frac{1}{q+1}-q+q_{1}+\frac{1}{q+1}<0 .
$$

Напомним, что $l$ - это число вершин в множестве $(P \cap S) \backslash\{v\}$, то есть число $u_{i}$. Известно, что $q_{1} \geqslant 1$. Поэтому

$$
l^{\prime}(q+1)+l<(q-1)(q+1)-1,
$$

то есть

$$
(q+1) l^{\prime}+l \leqslant q^{2}-3 .
$$

Очевидным образом из неравенства (9) получаем неравенство

$$
l \leqslant q^{2}-3
$$

и неравенство

$$
l^{\prime} \leqslant q-2
$$

Обозначим через $b_{j}$ (см. рис. 10$), j \in\{1, \ldots, r\}$, все вершины из множества $R \cap V(\tilde{I})$, в которые ведет ребро из вершины $v$ (множество $\{1, \ldots, r\}$ может быть пустым). Разберем несколько случаев.

1. $r \geqslant q^{2}-3$. Обозначим через $C_{v}$ объединение всех компонент связности графа $\widetilde{\mathscr{G}}(R)$, содержащих все вершины $b_{j}$, где $j \in\{1, \ldots, r\}$. Как отмечалось ранее, между множествами $P$ и $R^{\prime}$ не может быть ребер, значит, $C_{v} \subseteq R \backslash R^{\prime}$. В силу (8), справедливо равенство $d_{\tilde{I}}\left(w_{j}\right)=d$, где $j \in\{1, \ldots, m\}$. Таким образом, вершины из множества $P \cap R$ не смежны с вершинами $Z \backslash P$, а значит,

$$
C_{v} \subseteq\left\{w_{1}, w_{2}, \ldots, w_{m}\right\}
$$

Рассмотрим в множестве вершин $S$ графа $\widetilde{\mathscr{G}}$ все вершины, смежные с какой-нибудь вершиной из $C_{v}$. Обозначим множество всех таких вершин через $W$. Очевидно, что в силу равенств $d_{\tilde{I}}\left(w_{i}\right)=d$ и $d_{\varphi}\left(w_{i}\right)=d$, во-первых, $W \subseteq V(\tilde{I})$, во-вторых, $v \in W$ и, в-третьих, для всех вершин $u \in C_{v}$, верно равенство $d_{\widetilde{\varphi}\left(W \cup C_{v}\right)}(u)=d$.

1.1. $|W| \geqslant q^{2}-1$. Тогда

$$
\left|W \cap S^{\prime}\right|=|W|-|W \cap P \cap S| \geqslant q^{2}-1-(l+1) .
$$

Очевидно, что

$$
l^{\prime} \geqslant\left|W \cap S^{\prime}\right|
$$

Таким образом,

$$
l^{\prime} \geqslant q^{2}-2-l
$$




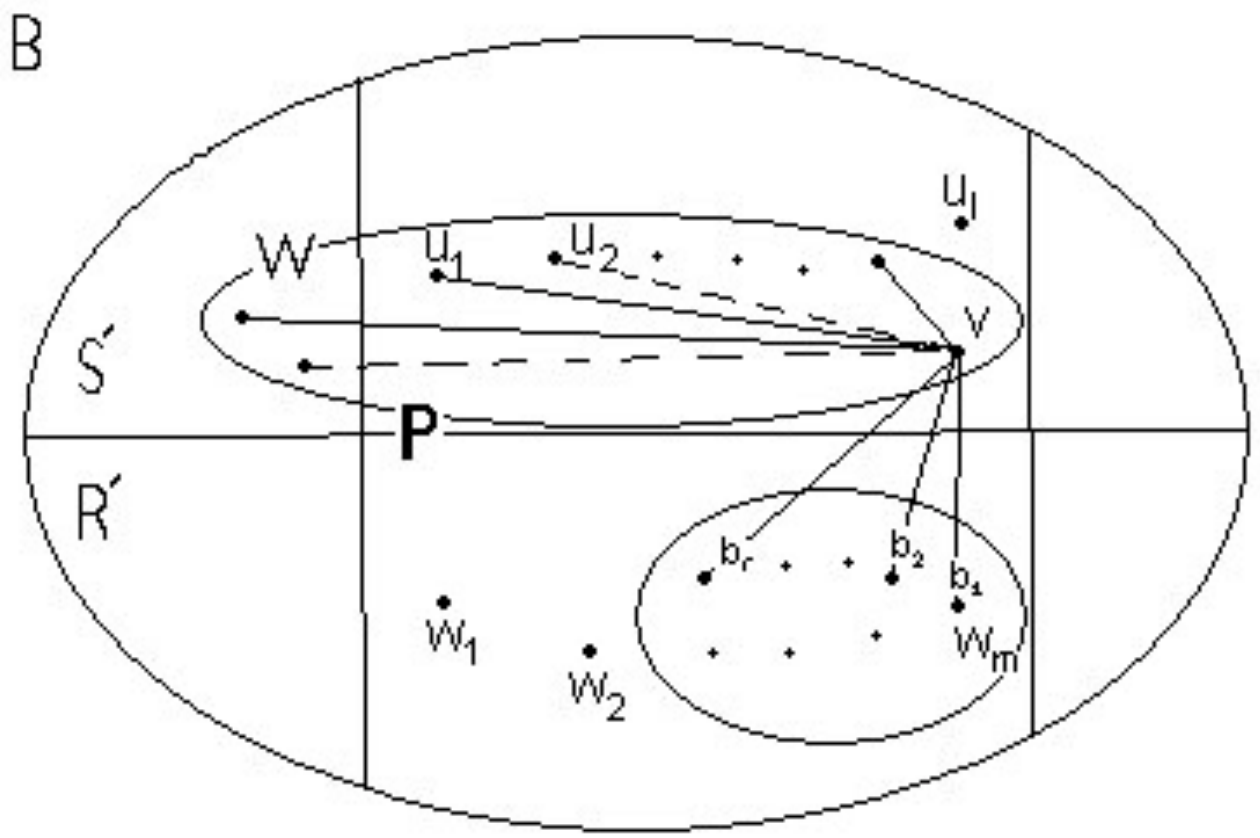

Рис. 10.

то есть

$$
l^{\prime}+l \geqslant q^{2}-2
$$

Получаем противоречие с неравенством (7).

Таким образом, мы показали, что

$$
|W| \leqslant q^{2}-2 .
$$

1.2. $|W| \leqslant q^{2}-2$. Проведем в графе $H$ дополнительно все ребра вида $(u, v)$, где $u \in W$ и $(u, v) \notin E(H)$. Получим новый граф $\Theta$. Проверим теперь, что все условия леммы 1 выполнены для графа $\hat{H}=\Theta\left(V(\Theta) \backslash C_{v}\right)$ с множествами $\hat{A}=A, \hat{B}=B \backslash C_{v}$ и величиной $\hat{d}=d$.

Условие 1 очевидно в силу того, что множество $A$ осталось прежним.

Условие 2 очевидно в силу того, что вершины из множества $C_{v}$ не были смежны ни с одной вершиной из множества $A$.

Что касается условия 3, неравенство (1) достаточно проверить только для вершин из множества $B$, для которых проводились новые ребра с концами в этих вершинах, то есть проверку нужно проводить для всех вершин из множества $W$. По определению, $\widehat{\mathscr{G}}=\hat{H}(\widehat{B})$. Для любой вершины $u \in W, u \neq v$ было добавлено к графу $\mathscr{G}$ максимум одно ребро, инцидентное $u$, но с другой стороны мы убрали по крайней мере одно ребро, ведущее в множество $C_{v}$ (по определению, $W-$ множество всех вершин из $S$, смежных с какой-нибудь вершиной из $C_{v}$ ). Таким образом, для таких вершин $u \in W$, что $u \neq v$, неравенство (1) осталось верным. Для вершины $v$ неравенство (1) будет выполнено 
потому, что $|W| \leqslant q^{2}-2$, значит, мы добавили не более, чем $q^{2}-3$ ребра. С другой стороны, множество $C_{v}$ по определению содержит все вершины $b_{j}$, где $j \in\{1, \ldots, r\}$, $r \geqslant q^{2}-3$, а значит, мы выкинули по крайней мере $q^{2}-3$ ребра с концом в $v$.

Было предположено, что $H$ - минимальный по числу вершин граф, для которого не выполнена лемма 1, поэтому лемма 1 выполнена для графа $\hat{H}$, в котором меньше вершин, чем в $H$. Поэтому можно правильным образом покрасить граф $\widehat{\mathscr{G}}$ в $d$ цветов так, чтобы для любой вершины $u \in A$ среди ее соседей в $\widehat{B}$ было не меньше $q$ вершин различных цветов. Обозначим такую раскраску $\xi$. Легко видеть, что все предположения из формулировки предложения 9 выполнены для $\Phi=\Theta\left(W \cup C_{v}\right)$, множеств $F_{1}=W$ и $F_{2}=C_{v}, D=d$ и вершины $v$. Рассмотрим правильную раскраску $\xi(W)$ графа $\Theta(W)$ в $d$ цветов. Согласно предложению 9 , раскраску $\xi(W)$ можно продолжить до правильной раскраски $\zeta$ в $d$ цветов графа $\Theta\left(W \cup C_{v}\right)$. Заметим, что в $\Theta$ нет ребер между множеством вершин $C_{v}$ и множеством вершин $V(H) \backslash\left(W \cup C_{v}\right)$, поэтому раскраски $\xi$ и $\zeta$ можно объединить в одну правильную раскраску графа $\mathscr{G}$ в $d$ цветов, кроме того, очевидно, что для любой вершины $u \in A$ в этой раскраске среди ее соседей в $B$ будет по крайней мере $q$ вершин различных цветов. Таким образом, мы получили нужную в лемме 1 раскраску для графа $H$, что противоречит условию утверждения 10.

2. $r \leqslant q^{2}-4$. В этом случае в графе $\tilde{I}$ из вершины $v$ ведет не более, чем $q^{2}-4$ ребра, в множество вершин $R \cap V(\tilde{I})$. Степень вершины $v$ в графе $\tilde{I}$ равна $d-q+q_{1}$. Значит, из вершины $v$ ведет как минимум $d-q+q_{1}-r-l^{\prime}$ ребер в множество вершин $\left\{u_{1}, u_{2}, \ldots, u_{l}\right\}$.

Заметим, что если у какой-то вершины $u \in S$ в графе $\widetilde{\mathscr{G}}$ есть ребро кратности $k$, то справедливо неравенство $d_{A}(u) \geqslant k-1$. В вершины $u_{1}, u_{2}, \ldots, u_{l}$ из $v$ идет по крайней мере $q^{3}+2 q^{2}-q-8-q+q_{1}-r-l^{\prime}$ ребер. Обозначим через $d_{i}$ кратность ребра $\left(v, u_{i}\right)$ в графе $\tilde{I}$ для всех $i \in\{1, \ldots, l\}$. Известно, что

$$
\sum_{i=1}^{l} d_{i} \geqslant q^{3}+2 q^{2}-2 q-8+q_{1}-r-l^{\prime}
$$

Отсюда получаем, что

$$
\sum_{i=1}^{l} d_{A}\left(u_{i}\right) \geqslant \sum_{i=1}^{l}\left(d_{i}-1\right) \geqslant q^{3}+2 q^{2}-2 q-8+q_{1}-r-l^{\prime}-l .
$$

Подставив неравенство (7) и равенство (8) в неравенство (3), получим, что

$$
l^{\prime}-q+q_{1}+\frac{1}{q+1}+\sum_{i=1}^{l}\left(d_{\tilde{I}}\left(u_{i}\right)-L\left(u_{i}\right)\right)<0 .
$$

Мы знаем, что

$$
d_{\tilde{I}}\left(u_{i}\right)-L\left(u_{i}\right) \geqslant d-d_{\varphi}\left(u_{i}\right)-\frac{d_{A}\left(u_{i}\right)}{q+1}
$$

для всех $i \in\{1, \ldots, l\}$. Применив неравенство (1), получаем, что

$$
d-d_{\varphi}\left(u_{i}\right)-\frac{d_{A}\left(u_{i}\right)}{q+1} \geqslant\left\lceil\frac{d_{A}\left(u_{i}\right)}{q}\right\rceil-\frac{d_{A}\left(u_{i}\right)}{q+1} \geqslant d_{A}\left(u_{i}\right) \frac{1}{q(q+1)} .
$$


Таким образом,

$$
d_{\tilde{I}}\left(u_{i}\right)-L\left(u_{i}\right) \geqslant d_{A}\left(u_{i}\right) \frac{1}{q(q+1)} .
$$

Подставив (14) в (13), получим, что

$$
l^{\prime}-q+q_{1}+\frac{1}{q+1}+\frac{1}{q(q+1)} \sum_{i=1}^{l} d_{A}\left(u_{i}\right)<0 .
$$

Подставив (12) в (15), получим, что

$$
l^{\prime}-q+q_{1}+\frac{1}{q+1}+\frac{q^{3}+2 q^{2}-2 q-8+q_{1}-r-l^{\prime}-l}{q(q+1)}<0 .
$$

Известно, что $l^{\prime} \geqslant 0$ и $q_{1} \geqslant 1$. Таким образом,

$$
l^{\prime}\left(1-\frac{1}{q(q+1)}\right)-(q-1)+\frac{1}{q+1}+\frac{q^{3}+2 q^{2}-2 q-8+1-r-l}{q(q+1)}<0 .
$$

Известно, что $r \leqslant q^{2}-4$, и $l \leqslant q^{2}-3$ в силу неравенства (10). Поэтому

$$
\frac{1}{q+1}+\frac{q^{3}+2 q^{2}-2 q-8+1-\left(q^{2}-4\right)-\left(q^{2}-3\right)-\left(q^{3}-q\right)}{q(q+1)}<0,
$$

то есть

$$
\frac{1}{q+1}-\frac{q}{q(q+1)}<0
$$

получили противоречие.

Таким образом, мы доказали неравенство (3) для графа $\tilde{I}$, а значит, доказано и предложение (10).

Вернемся к доказательству леммы 1. Для конкретного набора множеств $\lambda \in \Lambda$ начнем рекурсивно удалять, пока это возможно, вершины из графа $\widetilde{\mathscr{G}}$. Так как граф $\widetilde{\mathscr{G}}$ не рекурсивен, в конце от графа $\widetilde{\mathscr{G}}$ останется граф $\widetilde{\mathscr{G}}\left(S^{\prime} \cup R^{\prime}\right)$, где $S^{\prime} \subseteq S, R^{\prime} \subseteq R$. Выберем среди всех наборов из $\Lambda$ такой набор $\lambda$, что величина $\left|S^{\prime}\right|+\left|R^{\prime}\right|$ для него принимает минимальное значение. Заметим, что для множеств $S^{\prime}$ и $R^{\prime}$ выполнены все предположения предложения 10. Единственное неочевидное место в проверке этих условий - проверка неравенства (2).

Из графа $\widetilde{H}^{\prime}=\tilde{\mathscr{G}}\left(S^{\prime} \cup R^{\prime}\right)$ больше ничего нельзя рекурсивно удалить, поэтому в нем степени всех вершин не меньше $d$. По неравенству (1)

$$
L(u)=d_{\varphi}(u)+\frac{d_{A}(u)}{q+1} \leqslant d
$$

для всех вершин $u \in B$, причем $L(u)<d$ для всех вершин $u$ из множества $S$. Множество $S^{\prime}$ очевидно не пусто, поэтому

$$
\sum_{u \in \widetilde{H}^{\prime}} d_{\widetilde{H}^{\prime}}(u) \geqslant d\left|\tilde{H}^{\prime}\right|>\sum_{u \in \widetilde{H}^{\prime}} L(u) .
$$


Будем применять предложение 10 к множествам $S^{\prime}$ и $R^{\prime}$ до тех пор, пока не получим такой вершины, что ее степень в $\tilde{H}^{\prime}$ меньше $d$ (заметим, что мы не можем делать бесконечное число регулярных невырожденных сдвигов относительно множества $S^{\prime} \cup R^{\prime}$, так как при каждом таком сдвиге величина $\sum_{i=1}^{|A|}\left|S_{i} \cap S^{\prime}\right|$ уменьшается). Заметим, что в силу предложения 10 мы по-прежнему сможем рекурсивно удалить все вершины из $B$, кроме вершин $S^{\prime} \cup R^{\prime}$, а тогда мы сможем рекурсивно удалить еще и эту вершину степени, меньшей $d$. Получаем противоречие с тем, что мы выбрали набор с минимальной величиной $\left|S^{\prime}\right|+\left|R^{\prime}\right|$. Значит, найдется такой допустимый набор, что граф $\widetilde{\mathscr{G}}$ для этого набора будет рекурсивным. Таким образом, мы доказали лемму 1 и, следовательно, теорему 1 (см. замечание 7).

\section{Список литературы}

1. Bondy J. A., Murty U. S. R., Graph theory with applications. Elsevier, New York, 1976.

2. Hong-Jian L., Montgomery B., Poon Hoifung, Upper bounds of dynamic chromatic number. Ars Combinatoria (2003) 68, 193-201.

3. Montgomery B., Dynamic colouring, Ph. D. Dissertation. West Virginia Univ., 2001.

4. Brooks R. L., On colouring the nodes of network. Proc. Cambridge Philos. Soc. (1941) 37, 194-197.

5. Meng X., Miao L., Li Z. R., Su B., The conditional colouring numbers of pseudo-Halin graphs. Ars Combinatoria (2006) 79, 3-9.

6. Fan Suohai, Lai Hong-Jian, Lin Jianliang, Montgomery B., Tao Zhishui, Conditional colourings of graphs. Discrete Math. (2006) 16, 1997-2004.

7. Hind H., Molloy M., Reed B., Colouring a graph frugally. Combinatorica (1997) 17, 469-482.

8. Godsil C., Royle G., Algebraic graph theory. Springer, Berlin, 2001.

9. Lovász L., On decomposition of graphs. Stud. Sci. Math. Hungar. (1966) 1, 237-238.

Статья поступила 22.12.2007. Переработанный вариант поступил 10.06.2008. 